\title{
STRUCTURAL STABILITY OF VECTOR OPTIMIZATION PROBLEMS
}

\author{
by
}

Paulo Mbunga

Institut für Mathematik

Humboldt-Universität zu Berlin

\begin{abstract}
We study global stability properties for vector optimization problems of the type:

$\mathcal{V O P}(f, H, G)$ :

$\min \left\{f(x)=\left(f_{1}(x), \ldots, f_{l}(x)\right) \mid x \in M[H, G]\right\}$

where

$$
M[H, G]:=\left\{x \in \mathbb{R}^{n} \mid h_{i}(x)=0, \quad g_{j}(x) \leq 0, \quad i \in I, j \in J\right\}
$$

with

$$
I:=\{1, \ldots, m\}, \quad J:=\{1, \ldots, s\}, \quad L:=\{1, \ldots, l\} .
$$

We extend Guddat/Jongen's [5] concept of structural stability of scalar nonlinear optimization problems to vector optimization problems. Under the assumption that $M[H, G]$ is compact we prove the necessary condition for the structural stability of a vector optimization problem, i.e. the scalar problem

$\mathcal{P}^{\max }(f, H, G)$ :

$$
\min \left\{\max _{l \in L} f_{l}(x) \mid x \in M[H, G]\right\}
$$
\end{abstract}

has to be structurally stable.

\section{Introduction}

We consider the vector optimization problem

$\mathcal{V O} \mathcal{P}(f, H, G): \quad \min \left\{f(x)=\left(f_{1}(x), \ldots, f_{l}(x)\right) \mid x \in M[H, G]\right\}$,

where

$$
M[H, G]:=\left\{x \in \mathbb{R}^{n} \mid h_{i}(x)=0, \quad g_{j}(x) \leq 0, \quad i \in I, j \in J\right\}
$$

Key words and phrases. - vector optimization, structural stability, strong stability, constraint qualification (Mangasarian-Fromovitz).

This work was supported by the Graduiertenkolleg Geometrie und Nichlineare Analysis of the Humboldt-Universität. 
with

$$
I:=\{1, \ldots, m\}, \quad J:=\{1, \ldots, s\}, \quad L:=\{1, \ldots, l\} .
$$

Unless otherwise statet, we will assume that the functions $f_{k}, g_{j}, k \in L, j \in J$ belong to $C^{2}\left(\mathbb{R}^{n}, \mathbb{R}\right)$. The lower level set corresponding to $u \in \mathbb{R}^{|L|}$ will be denoted as follows:

$$
\mathcal{L}^{u}(f, H, G):=\{x \in M[H, G] \mid f(x) \leq u\}, \quad u \in \mathbb{R}^{|L|} .
$$

The partial ordering $\leq$ is induced by a convex cone $D$ with

$$
x \leq y \quad: \Longleftrightarrow y-x \in D .
$$

Definition 1. - The vector optimization problems $\mathcal{V O P}(f, H, G)$ and $\mathcal{V O P}(\tilde{f}, \tilde{H}, \tilde{G})$ are called equivalent if there exist continuous mappings $\phi: \mathbb{R}^{|L|} \times \mathbb{R}^{n} \longrightarrow \mathbb{R}^{n}$ and $\psi: \mathbb{R}^{|L|} \longrightarrow \mathbb{R}^{|L|}$ with the properties $P 1-P 3$ :

$P 1$ For every $u \in \mathbb{R}^{|L|}$ the mapping $\phi_{u}: \mathbb{R}^{n} \longrightarrow \mathbb{R}^{n}$ is a homeomorphism from $\mathbb{R}^{n}$ onto itself, where $\phi_{u}:=\phi(u, x)$.

P2 The mapping $\psi$ is a homeomorphism from $\mathbb{R}^{|L|}$ onto itself and $\psi$ is $D$ monotonically increasing; i.e.

$$
q_{1} \underset{D}{\leq} q_{2} \text { implies } \psi\left(q_{1}\right) \underset{D}{\leq} \psi\left(q_{2}\right) \text { for } q_{1}, q_{2} \in \mathbb{R}^{|L|} .
$$

P3 $\phi_{u}\left[\mathcal{L}^{u}(f, H, G)\right]=\mathcal{L}^{\psi(u)}(\tilde{f}, \tilde{H}, \tilde{G})$ for all $u \in \mathbb{R}^{|L|}$.

Definition 2. - The vector optimization problems $\mathcal{V O P}(f, H, G)$ is called structurally stable if there exists a $C_{S}^{2}$-neighborhood $\mathcal{O}$ of $(f, H, G)$ with the property that $\mathcal{V O P}(f, H, G)$ and $\mathcal{V O P}(\tilde{f}, \tilde{H}, \tilde{G})$ are equivalent for all $(\tilde{f}, \tilde{H}, \tilde{G}) \in \mathcal{O}$.

The $C_{S}^{2}$-topology above for the product $\prod_{i=1}^{r} C^{2}\left(\mathbb{R}^{n}, \mathbb{R}\right)$ will be the product topology generated by the strong (or Whitney-) $C^{2}$-topology $C_{S}^{2}$ on each factor $C^{2}\left(\mathbb{R}^{n}, \mathbb{R}\right.$ ) (cf. [6],[9]). A typical base-neighbourhood of a function $\rho \in C^{2}\left(\mathbb{R}^{n}, \mathbb{R}\right)$ is the set $\rho+\mathcal{W}_{\epsilon}$, where $\mathcal{W}_{\epsilon}$ is defined as follows with the aid of a continuous positive $\epsilon: \mathbb{R}^{n} \longrightarrow \mathbb{R}$

(4) $\mathcal{W}_{\epsilon}=\left\{\eta \in C^{2}\left(\mathbb{R}^{n}, \mathbb{R}\right)|| \eta(x)\left|+\sum_{i}\right| \frac{\partial \eta}{\partial x_{i}}(x) \mid+\right.$

$$
\left.+\sum_{i, j}\left|\frac{\partial^{2} \eta}{\partial x_{i} \partial x_{j}}(x)\right|<\epsilon(x), \forall x \in \mathbb{R}^{n}\right\} .
$$

We have the chance of a more local control for data corresponding to the scalar problem $\mathcal{P}(r, H, G), r \in C^{2}\left(\mathbb{R}^{n}, \mathbb{R}\right)$ by putting for any subset $U$ of $\mathbb{R}^{n}$ :

(5) $\operatorname{norm}[(r, H, G), U]=$

$$
\sup _{x \in U} \max _{\eta \in\left\{r, h_{i}, i \in I, g_{j}, j \in J\right\}}\left\{|\eta(x)|+\sum_{i}\left|\frac{\partial \eta}{\partial x_{i}}(x)\right|+\sum_{i, j}\left|\frac{\partial^{2} \eta}{\partial x_{i} \partial x_{j}}(x)\right|\right\} .
$$


Remark 1. - If $|L|=1$ and $D=\mathbb{R}_{+}^{|L|}$, then we have the structural stability for the scalar nonlinear problem introduced in [5].

We consider the following scalar optimization problem

$$
\mathcal{P}^{\max }(f, H, G): \quad \min \left\{\max _{l \in L} f_{l}(x) \mid x \in M[H, G]\right\} .
$$

For the corresponding lower level set we shall use the notation

$$
\mathcal{L}_{\text {max }}^{t}(f, H, G):=\left\{x \in M[H, G] \mid \max _{l \in L} f_{l}(x) \leq t\right\}, \quad t \in R .
$$

We use the notations given in Weber [14] and denote the canonical projection of the space $\mathbb{R}^{n+1}$ on the first $n$ components or on the last component by $\operatorname{pr}_{(1, \ldots, n)}$ and $p r_{n+1}$, respectively; i.e.

$$
\check{x}=\left(\operatorname{pr}_{(1, \ldots, n)}(\check{x}), p r_{n+1}(\check{x})\right)=\left(x, x_{n+1}\right) \in \mathbb{R}^{n} \times \mathbb{R} .
$$

Let us now look at the following equivalent differentiable optimization problem which in particular reveals an objective function of class $C^{2}$.

$\mathcal{P}^{\vee}(\check{f}, \check{H}, \check{G}): \quad \min \left\{\check{f}(\check{x}):=\operatorname{pr}_{n+1}(\check{x}) \mid x \in M[\check{H}, \check{G}]\right\}$,

where

$$
M[\check{H}, \check{G}]:=\left\{\left(x, x_{n+1}\right) \in \mathbb{R}^{n+1} \mid x \in M[H, G], f_{l}(x)-x_{n+1} \leq 0 \quad(l \in L)\right\},
$$

namely with $\check{h}_{i}(\check{x}):=h_{i}(x)(i \in I)$ and

$$
\check{g}_{j}(\check{x}):=\left\{\begin{array}{l}
g_{j}(x), \text { for } j \in J \\
f_{l}(x)-x_{n+1}, \text { for } j=s+l, \quad l \in L .
\end{array}\right.
$$

$\mathcal{P}^{\vee}(\check{f}, \check{H}, \check{G})$ is strongly related to scalarization methods for vector optimization problems. As pointed out in Weidner [16], this scalarization method includes the known procedures of Brosowski and Conci [3], Helbig [8], Wierzbicki [17] and other papers cited in [7]. Roughly speaking, each minimal point of $\mathcal{P}^{\vee}(\check{f}, \check{H}, \check{G})$ is weakly efficient and vice versa each weakly efficient and hence each efficient point solves the scalar problem $\mathcal{P}^{\vee}(\check{f}, \check{H}, \check{G})$.

We note that the lower level sets $\mathcal{L}_{\text {max }}^{t}(f, H, G)$ of $\mathcal{P}^{\max }(f, H, G)$ are just the projection in the sense of $\operatorname{pr}_{(1, \ldots, n)}$ of the corresponding level sets $\mathcal{L}^{\vee t}(\check{f}, \check{H}, \check{G})$ due to $\mathcal{P}^{\vee}(\check{f}, \check{H}, \check{G})$. Both sets are homotopy-equivalent (cf. Jongen et al. [9], Lemma 4.2.1) and $M[\check{H}, \check{G}]$ is just the part of the epigraph $E\left(\max _{l \in L} f_{l}\right)$ over the feasible set $M[H, G]$.

Definition 3. - The Linear Independence Constraint Qualification (shortly LICQ) is said to hold at $x \in M[H, G]$ if the vectors $D h_{i}(x), i \in I, D g_{j}(x), j \in J_{o}(x)$, are linearly independent. The Mangasarian-Fromovitz Constarint Qualification (shortly $M F C Q)$ is said to hold at $x \in M[H, G]$ if the following conditions MF1 and MF2 are satisfied:

MF1 The vectors $D h_{i}(x), i \in I$ are linearly independent. 
$M F 2$ There exists a vector $\xi \in \mathbb{R}^{n}$ satisfying:

$$
\begin{aligned}
D h_{i}(x) \xi=0, & i \in I \\
\left.D g_{j}(x)\right) \xi \leq 0, & j \in J_{o}(x) .
\end{aligned}
$$

$A$ vector $\xi$ satisfying (6) and (7) will be called an MF-vector.

For $x \in \mathbb{R}^{n}$ and $\rho>0$ let $B(x, \rho)$ denote the Euclidean ball in $\mathbb{R}^{n}$, centered at $x$ with radius $\rho$. Following M. Kojima $([\mathbf{1 1}])$ we define:

Definition 4. - Let $x^{u} \in M[H, G]$ be a Kuhn-Tucker point for $\mathcal{P}(r, H, G), r \in$ $C^{2}\left(\mathbb{R}^{n}, \mathbb{R}\right)$. Then $x^{u}$ is called strongly stable if for some $\bar{\delta}>0$ and each $\delta \in(0, \bar{\delta}]$ there exists an $\alpha>0$ such that whenever $(\tilde{r}, \tilde{H}, \tilde{G})$ satisfies norm $[(r-\tilde{r}, H-\tilde{H}, G-$ $\left.\tilde{G}), B\left(x^{u}, \delta\right)\right] \leq \alpha$, the ball $B\left(x^{u}, \delta\right)$ contains a Kuhn-Tucker point for $\mathcal{P}(\tilde{r}, \tilde{H}, \tilde{G})$ which is unique in $B\left(x^{u}, \delta\right)$.

Lemma 1 (Kojima [11]). - Let $\bar{x}^{u} \in M[H, G]$ be a Kuhn-Tucker point for $\mathcal{P}(r, H, G), r \in C^{2}\left(\mathbb{R}^{n}, \mathbb{R}\right)$.

1. If LICQ is satisfied at $\bar{x}^{u}$, then $\bar{x}^{u}$ is a strongly stable Kuhn-Tucker point if and only if the matrix $D^{2} L_{[\lambda, \mu]}\left(\bar{x}^{u}\right)$ has nonvanishing determinants with a common sign on the subspace $W\left(\bar{x}^{u}, \bar{J}\right)$ for all $\bar{J}$ with $J_{+}\left(\bar{x}^{u}\right) \subset \bar{J} \subset J_{o}\left(\bar{x}^{u}\right)$, where

$$
\begin{aligned}
J_{+}\left(\bar{x}^{u}\right) & =\left\{j \in J_{o}\left(\bar{x}^{u}\right) \mid \mu_{j}>0\right\} \\
W\left(\bar{x}^{u}, \bar{J}\right) & =\left\{\xi \in \mathbb{R}^{n} \mid D h_{i}\left(\bar{x}^{u}\right) \xi=0, i \in I, D g_{j}\left(\bar{x}^{u}\right) \xi=0, j \in \bar{J}\right\} \\
L_{[\lambda, u]}(x) & :=r(x)+\sum_{i \in I} \lambda_{i} h_{i}(x)+\sum_{j \in J_{o}\left(\bar{x}^{u}\right)} u_{j} g_{j}(x) .
\end{aligned}
$$

2. Let MFCQ be satisfied at $\bar{x}^{u}$, but LICQ not. Then, $\bar{x}^{u}$ is a strongly stable KuhnTucker point if and only if for every $(\lambda, \mu)$ the matrix $D^{2} L_{[\lambda, \mu]}\left(\bar{x}^{u}\right)$ is positive definite on the subspace $W\left(\bar{x}^{u}, J_{+}\left(\bar{x}^{u}\right)\right)$.

Firstly we note that the structural stability of the problem $\mathcal{P}^{\max }(f, H, G)$ was characterized in Weber [14].

Theorem 1 (Main result). - Let $D=\mathbb{R}_{+}^{|L|}$ and the vector optimization problem $\mathcal{V O P}(f, H, G)$ with compact feasible set $M[H, G]$ be structurally stable. Then the problem $\mathcal{P}^{\max }(f, H, G)$ is structurally stable.

\section{Lemmas and preliminary results}

Lemma 2. - Let $t \in \mathbb{R}, D=\mathbb{R}_{+}^{|L|}$ and $u:=(t, \ldots, t)=t e, e=(1, \ldots, 1) \in \mathbb{R}^{|L|}$. Then the following relations between lower level sets are fulfilled:

$$
\mathcal{L}_{\max }^{t}(f, H, G)=\operatorname{pr}_{(1, \ldots, n)} \mathcal{L}^{\vee t}(\check{f}, \check{H}, \check{G})=\mathcal{L}^{u}(f, H, G) .
$$

Proof. - The first equality is obvious. We show the second equality. $\mathcal{L}^{u}(f, H, G) \subseteq \operatorname{pr}_{(1, \ldots, n)} \mathcal{L}^{\vee t}(\check{f}, \check{H}, \check{G})$ : Let $x \in \mathcal{L}^{u}(f, H, G)$. Then $x \in M[H, G]$, $f_{l}(x) \leq t, l \in\{1, \ldots, L\}$. This implies that $(x, t) \in M(\check{H}, \check{G})$ and so $x \in$ $\operatorname{pr}_{(1, \ldots, n)} \mathcal{L}^{\vee t}(\check{f}, \check{H}, \check{G})$. 
$\mathcal{L}^{u}(f, H, G) \supseteqq p r_{(1, \ldots, n)} \mathcal{L}^{\vee t}(\check{f}, \check{H}, \check{G}):$ Let $x \in \operatorname{pr}_{(1, \ldots, n)} \mathcal{L}^{\vee t}(\check{f}, \check{H}, \check{G})$. Then, there exists a $z \in \mathbb{R}$ with $(x, z) \in \mathcal{L}^{\vee t}(\check{f}, \breve{H}, \check{G})$. This implies that $x \in M[H, G], f_{l}(x) \leq z$, $l \in L$ and $z \leq t$ and so $f_{l}(x) \leq t, l \in L$. That means $x \in \mathcal{L}^{u}(f, H, G)$.

Lemma 3. - Let D be a pointed, closed and convex cone. Then there exists a $\bar{u} \in$ $\mathbb{R}^{|L|}$ with:

$$
u_{1} \underset{D}{ } \bar{u}, \quad u_{2} \underset{D}{\bar{u}}
$$

for all $u_{1}, u_{2} \in \mathbb{R}^{|L|}$.

Proof. - We follow the idea by Tanino in [12] (Proof of the Theorem 3.3.1). Pick

$$
d \in \text { int } D
$$

and define

$$
y(\alpha)=\alpha d \quad \alpha \in \mathbb{R} .
$$

We claim that, for any $y \in \mathbb{R}^{|L|}$, there exists $\alpha>0$ such that

$$
y \in y(\alpha)-D .
$$

If it is not true, then applying the standard separation theorem to the ray $\{y-\alpha d$ : $\alpha>0\}$ and the convex cone $-D$, we deduce the existence of $\nu \in \mathbb{R}^{p} \backslash\{0\}$ such that

$$
\langle\nu, y-\alpha d\rangle \geq 0 \quad \text { for all } \alpha>0
$$

and

$$
\left\langle\nu, d^{\prime}\right\rangle \geq 0 \quad \text { for all } d^{\prime} \in D .
$$

Since $d \in$ int $D,\langle\nu, d\rangle>0$ from the latter inequality and so

$$
\langle\nu, y-\alpha d\rangle<0
$$

for sufficiently large $\alpha$, which is a contradiction.

We can therefore choose $\alpha_{1}, \alpha_{2}>0$ with

$$
u_{1} \in \alpha_{1} d-D \quad \text { and } u_{2} \in \alpha_{2} d-D .
$$

Take $\alpha_{\max }:=\max \left\{\alpha_{1}, \alpha_{2}\right\}$. Since $\alpha_{i} d \leq \alpha_{\max } d, i=1,2$, we can observe that the inequalities (9) are satisfied by $\bar{u}:=\alpha_{\max } d$.

\section{Proof of the Necessity Part}

Let us now assume that the problem $\mathcal{V O P}(f, H, G)$ is structurally stable according to the Definition 2. Then from the results given by Weber in [10] (see also [14] and [5]) we have to verify the following conditions:

$(C 1)$ The Mangasarian-Fromovitz constraint qualification is satisfied at every point of $M[H, G]$.

(C2) Every Kuhn-Tucker point of $\mathcal{P}^{\max }(f, H, G)$ is strongly stable.

(C3) Different Kuhn-Tucker points have different $(f-)$ values. 
Proof of $(\mathrm{C} 1)$ : We show that the structural stability of $\mathcal{V O P}(f, H, G)$ implies (C1). Consider a $C_{s}^{2}$-neighborhood $\mathcal{O}$ of $(f, H, G)$ with the property: for every $(\tilde{f}, \tilde{H}, \tilde{G}) \in \mathcal{O}$ the set $M[\tilde{H}, \tilde{G}]$ is compact and moreover $\mathcal{V O P}(f, H, G)$ is equivalent with $\mathcal{V O P}(\tilde{f}, \tilde{H}, \tilde{G})$. Choose an arbitrary element $(\tilde{f}, \tilde{H}, \tilde{G}) \in \mathcal{O}$. Let $\phi$ and $\psi$ be mappings which establish the equivalence according Definition 1. Take $\alpha, \tilde{\alpha} \in \mathbb{R}^{|L|}$ with

$$
\begin{array}{ll}
\alpha:=\left(\alpha_{1}, \ldots, \alpha_{|L|}\right), \quad \alpha_{i}:=\max _{x \in M[H, G]} f_{i}(x) \\
\tilde{\alpha}:=\left(\tilde{\alpha}_{1}, \ldots, \tilde{\alpha}_{|L|}\right), \quad \tilde{\alpha}_{i}:=\max _{x \in M[\tilde{H}, \tilde{G}]} \tilde{f}_{i}(x)
\end{array}
$$

and consider the vectors $\alpha$ and $\psi^{-1}(\tilde{\alpha})$. Lemma 9 implies the existence of a $\bar{u} \in \mathbb{R}^{|L|}$ such that $\alpha \underset{D}{\bar{u}}$ and $\psi^{-1}(\tilde{\alpha}) \underset{D}{\bar{u}}$. We note that $\mathcal{L}^{\bar{u}}(f, H, G)=M[H, G]$. Since $\psi$ is monotonically increasing, we have $\tilde{\alpha} \underset{D}{\leq} \psi(\bar{u})$. Thus $\mathcal{L}^{\psi(\bar{u})}(\tilde{f}, \tilde{H}, \tilde{G})=M[\tilde{H}, \tilde{G}]$. From $\phi_{\bar{u}}\left(\mathcal{L}^{\bar{u}}(f, H, G)\right)=\mathcal{L}^{\psi(\bar{u})}(\tilde{f}, \tilde{H}, \tilde{G})$, we have $\phi_{\bar{u}}(M[H, G])=M[\tilde{H}, \tilde{G}]$. Hence, $\phi_{\bar{u}}$ establishes a homeomorphism between $M[H, G]$ and $M[\tilde{H}, \tilde{G}]$. So, we have shown: for every $(\tilde{H}, \tilde{G})$ in some $C_{S}^{2}$-neighborhood of $(H, G)$ the sets $M[H, G]$ and $M[\tilde{H}, \tilde{G}]$ are homeomorphic. Then, with the help of the Stability Theorem from [5] we may equivalently say that MFCQ is satisfied at every point $x \in M[H, G]$. Hence, the condition $(\mathrm{C} 1)$ is valid.

Proof of $(\mathrm{C} 2)$ : Let us assume that there is a stationary point which is not strongly stable, i.e. condition $(\mathrm{C} 2)$ is not valid. In order to arrive at a contradiction, the perturbation result from Weber [14] plays a central part.

Lemma 4 (Perturbation Lemma, Weber [14]). - Let $M[H, G]$ be compact and suppose that (C1) holds. Suppose further, that $\mathcal{P}^{\max }(V, H, G)$ has a Kuhn-Tucker point $\bar{x}^{u}$ which is not strongly stable. Then, there exist a number $k \in \mathbb{N}$ and functions $\left(V^{1}, \tilde{H}, \tilde{G}\right),\left(V^{2}, \tilde{H}, \tilde{G}\right)$, both arbitrarily $C_{S}^{2}$-near $(V, H, G)$, with the following properties:

(i) Problem $\mathcal{P}^{\max }\left(V^{1}, \tilde{H}, \tilde{G}\right)$ has $k$ Kuhn-Tucker points, exactly one of which is not strongly stable.

(ii) Problem $\mathcal{P}^{\max }\left(V^{2}, \tilde{H}, \tilde{G}\right)$ has at least $k+1$ Kuhn-Tucker points, all of them being strongly stable.

(iii) Conditions $C 1$ and $C 3$ hold for problems $\mathcal{P}^{\max }\left(V^{i}, \tilde{H}, \tilde{G}\right), i=1,2$.

For $r \in C^{2}\left(\mathbb{R}^{n}, \mathbb{R}\right)$ we denote the set $\{x \in M[H, G] \mid a \leq r(x) \leq b \in\}$ by $\mathcal{L}_{b}^{a}(r, H, G)$ for $a \leq b, a, b \in \mathbb{R}$.

The next Lemma is proved by Guddat and Jongen in [5].

Lemma 5. - Let the feasible set $M[H, G]$ corresponding to the problem $\mathcal{P}(r, H, G)$ be compact and suppose that Condition $C 1$ is fulfilled. Let $a, b \in \mathbb{R}$ be given with $a<b$.

(i) If $\mathcal{L}_{b}^{a}(r, H, G)$ contains no Kuhn-Tucker point, then $\mathcal{L}^{a}(r, H, G)$ and $\mathcal{L}^{b}(r, H, G)$ are homeomorphic. 
(ii) Suppose that $\mathcal{L}_{b}^{a}(r, H, G)$ contains exactly one Kuhn Tucker point $\bar{x}^{u}$ with a< $r\left(\bar{x}^{u}\right)<b$, and suppose that $\bar{x}^{u}$ is strongly stable. Then the sets $\mathcal{L}^{a}(r, H, G)$ and $\mathcal{L}^{b}(r, H, G)$ are not homeomorphic.

Lemma 6. - Let $M[H, G]$ be compact and suppose that Condition $C 1$ is fulfilled. Let $V^{1}, V^{2} \in C^{2}\left(\mathbb{R}^{n}, \mathbb{R}\right)^{|L|}$ be functions satisfying Condition C3 w.r.t. $\mathcal{P}^{\max }\left(V^{i}, H, G\right), i=1,2$, with the following additional properties:

(i) Problem $\mathcal{P}^{\max }\left(V^{1}, H, G\right)$ has $k$ Kuhn-Tucker points, exactly one of which is not strongly stable.

(ii) Problem $\mathcal{P}^{\max }\left(V^{2}, H, G\right)$ has at least $k+1$ Kuhn-Tucker points, all of them being strongly stable.

Then, the vector optimization problems $\mathcal{V O P}\left(V^{i}, H, G\right), i=1,2$ are not equivalent.

Proof. - We refer to the $n$-dimensional problems $\mathcal{P}^{\max }\left(V^{i}, H, G\right), i=1,2$ due to the corresponding $(n+1)$-dimensional problems $\mathcal{P}^{\vee}\left(\check{f}^{i}, \check{H}, \breve{G}\right), i=1,2$. For the two latter problems, we study the topological behaviour of the lower level sets, when the level parameter $t$ increasingly traverses the line of real numbers. Taking account of Condition $C 3$ formulated for both problems and the Lemma 5 , it follows that the homeomorphytype of the lower level set $\mathcal{L}^{\vee t}\left(\check{f}^{2}, \check{H}, \check{G}\right)$ changes at least $k+1$, and at least $k-1$, but (only) at most $k$ such changes for $\mathcal{L}^{\vee t}\left(\check{f}^{1}, \check{H}, \check{G}\right)$. The pairwise homotopy equivalence between the lower level sets in $\mathbb{R}^{n}$ and $\mathbb{R}^{n+1}$ gives rise to the analogous description in dimension $n$ of the topological behavior of $\mathcal{L}_{\text {max }}^{t}\left(V^{i}, H, G\right), i=1,2$. From Lemma 2 (8) it follows that $\mathcal{L}^{t e}\left(V^{i}, H, G\right), i=1,2$ have two different numbers for the changes of the topological type in $\mathbb{R}^{n}$, as we move along the line $u(t)=t e, e \in \mathbb{R}^{|L|}, t \in \mathbb{R}$. If the problems $\mathcal{V O P}\left(V^{i}, H, G\right), i=1,2$ would be equivalent, then these two numbers have to coincide.

We now apply the perturbational result for our indirect proof of Condition $C 2$. If this condition does not hold, we can apply Lemma 4 and Lemma 6 in order to obtain two non-equivalent problems $\mathcal{V O P}\left(f^{i}, \tilde{H}, \tilde{G}\right), i=1,2$ with each $\left(f^{i}, \tilde{H}, \tilde{G}\right)$ arbitrarily $C_{S}^{2}$-near to $(f, H, G)$. This is in contradiction with structural stability.

Proof of (C3): We know the validity of $C 1$ and $C 2$. If $\mathcal{P}^{\max }(f, H, G)$ does not satisfy Condition $C 3$, whenever the equation $\max _{l \in L} f\left(\bar{x}_{1}^{u}\right)=\max _{l \in L} f\left(\bar{x}_{2}^{u}\right)$ holds for two different Kuhn-Tucker points of $\mathcal{P}^{\max }(f, H, G)$ then an addition of one small positive and one small negative constant to all functions $f_{k} \quad(k \in L)$ locally at $\bar{x}_{1}^{u}$ and $\bar{x}_{2}^{u}$, respectively, leads to a problem $\mathcal{P}^{\max }(\bar{f}, H, G)$ satisfying Condition C3. Let $k$ the number of Kuhn-Tucker points for $\mathcal{P}^{\max }(\bar{f}, H, G)$. Then, reasoning as in the proof of Lemma 6, the homeomorphy-type of

$\mathcal{L}_{\text {max }}^{t}(\bar{f}, H, G)$ for increasing $t$, changes exactly $k$-times. The corresponding number of changes w.r.t. $\mathcal{P}^{\max }(f, H, G)$ is less than $k$. So, as in $\operatorname{Lemma} 6, \mathcal{V O P}(f, H, G)$ and $\mathcal{V O P}(\bar{f}, H, G)$ are not equivalent. This leads again to a contradiction with the condition on structural stability of $\mathcal{V O P}(f, H, G)$. 


\section{References}

[1] Bank, B.; Guddat, J.; Klatte, D.; Kummer, B. and Tammer, K.: Non-Linear Parametric Optimization. Akademie-Verlag. Berlin, 1982.

[2] Guddat, J., Jongen, H. Th., Rueckmann, J.: On stability and stationary points in nonlinear optimization, J. Aust. Math. Soc., Ser. B 28 (1986), 36 - 56.

[3] Brosowski, B. and Conci, A.: On vector optimization and Parametric Programming. Segundas Jornadas Latino Americanas de Matematica Aplicada, 2(1983), 483 - 495.

[4] Guddat, J.; Guerra Vasquez, F. and Jongen, H. Th.: Parametric Optimization, Singularities, Pathfollowing and Jumps. John Wiley, Chichester 1990.

[5] Guddat, Juergen and Jongen, H. Th.: Structural Stability in Nonlinear Optimization. In: Optimization 18 (1987)5, $617-631$.

[6] Hirsch, M. W.: Differential Topology, Springer-Verlag, Berlin, Heidelberg, New York, 1976.

[7] Helbig, S.: On Quatitative and Qualitative Stability of a Scalarization Method, I. In Parametric Optimization and Related Topics III, 255-270, Peter Lang Verlag, Franfurt am Main 1993.

[8] Helbig, S.: Approximation of the Efficient Point Set by Perturbation of the Ordering Cone, Zeitschrift für Operations Research 35(1991), $197-220$.

[9] Jongen, H. Th.; Jonker, P., Twilt, F.: Nonlinear Optimization in $\mathbb{R}^{n}$, I. Morse Theory, Chebishev approximation, Peter Lang Verlag, Frankfurt, 1983.

[10] Jongen, H. Th and Weber, G.-W. : Nonlinear Optimization: Characterization of Structural Stability. In: J. Global Optimization 1 (1991), $47-64$.

[11] Kojima, M.: Strongly stable stationary solutions in nonlinear programs. In: S.M. Robinson (ed.): Analysis and computation of fixed points. Academic Press, New York, 1980.

[12] Sawaragi Y., Nakayama H., Tanino T.: Theory of Multiobjective Optimization. Academic Press, Inc., Orlando 1985.

[13] The Luc, D.: Theory of Vector Optimization. Springer-Verlag, Berlin, 1989.

[14] Weber, G.: Minimization of a max-type function: Characterization of structural stability. In Parametric Optimization and Related Topics III, 519 - 538, Peter Lang Verlag, Franfurt am Main 1993.

[15] Weber, G.: Charakterisierung struktureller Stabilität in der nichtlinearen Optimierung. Aachener Beiträge zur Mathematik, Band 5, Aachen 1992.

[16] Weidner, P.: An approach to different scalarizations in vector optimization. Wissenschaftliche Zeitschrift der TH Ilmenau 3(1990), 103 - 110.

[17] Wierzbicki, A. P.: On the Completeness and Constructiveness of Parametric Characterization to Vector Optimization Problem, OR Spektrum 8(1986), 73 - 87.

[18] Wierzbicki, A. P.: Basic Properties of scalarizing functional for multiobjective optimization. Math. Oper.Stat. Optim. 8, 55-60, 1977.

\footnotetext{
Paulo Mbunga, Institut für Mathematik, Humboldt-Universität zu Berlin, Campus Adlershof, Rudower Chaussee 25, Haus II, 4. Etage D-12489 Berlin

E-mail : mbunga@mathematik.hu-berlin.de
} 